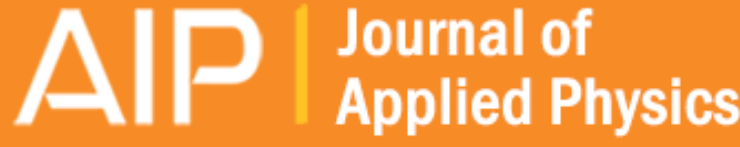

\section{Photoinduced spin-polarized current in InSb-based structures}

M. Frazier, J. G. Cates, J. A. Waugh, J. J. Heremans, M. B. Santos, X. Liu, and G. A. Khodaparast

Citation: Journal of Applied Physics 106, 103513 (2009); doi: 10.1063/1.3262496

View online: http://dx.doi.org/10.1063/1.3262496

View Table of Contents: http://scitation.aip.org/content/aip/journal/jap/106/10?ver=pdfcov

Published by the AIP Publishing

\section{Articles you may be interested in}

Anomalous circular photogalvanic effect of the spin-polarized two-dimensional electron gas in $\mathrm{Mg} 0.2 \mathrm{Zn} 0.8 \mathrm{O} / \mathrm{ZnO}$ heterostructures at room temperature

Appl. Phys. Lett. 102, 192405 (2013); 10.1063/1.4805079

Spin injection and circular polarized electroluminescence from InAs-based spin-light emitting diode structures J. Appl. Phys. 107, 114510 (2010); 10.1063/1.3354021

Generation of highly spin-polarized currents in cascaded InAs spin filters

J. Appl. Phys. 105, 093714 (2009); 10.1063/1.3124359

Type II transition in InSb-based nanostructures for midinfrared applications

J. Appl. Phys. 103, 114516 (2008); 10.1063/1.2938063

Ultrafast spin-polarized electrical currents injected in a strained zinc blende semiconductor by single color pulses Appl. Phys. Lett. 86, 061102 (2005); 10.1063/1.1855426

\section{MIT LINCOLN} LABORATORY CAREERS

Discover the satisfaction of innovation and service to the nation
- Space Control

- Air \& Missile Defense

- Communications Systems \& Cyber Security

- Intelligence, Surveillance and

Reconnaissance Systems
- Advanced Electronics

- Tactical Systems

- Homeland

Protection

- Air Traffic Control

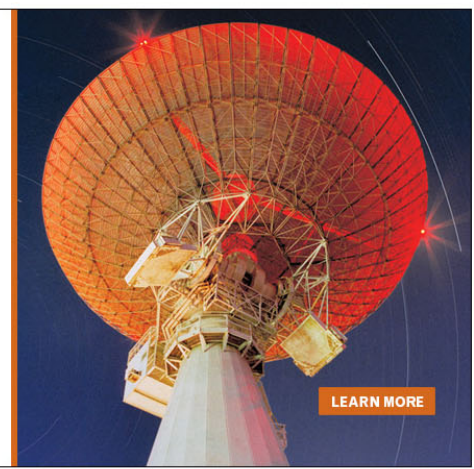




\title{
Photoinduced spin-polarized current in InSb-based structures
}

\author{
M. Frazier, ${ }^{1}$ J. G. Cates, ${ }^{1}$ J. A. Waugh, ${ }^{1}$ J. J. Heremans, ${ }^{1}$ M. B. Santos,${ }^{2}$ X. Liu, ${ }^{3}$ and \\ G. A. Khodaparast ${ }^{1, a)}$ \\ ${ }^{1}$ Department of Physics, Virginia Tech, Blacksburg, Virginia 24061, USA \\ ${ }^{2}$ Homer L. Dodge Department of Physics and Astronomy, University of Oklahoma, Norman, \\ Oklahoma 73019, USA \\ ${ }^{3}$ Department of Physics, University of Notre Dame, Notre Dame, Indiana 46556, USA
}

(Received 6 August 2009; accepted 13 October 2009; published online 20 November 2009)

\begin{abstract}
We demonstrate the observation of spin-polarized photocurrent in InSb films grown on GaAs and InP substrates and InSb quantum wells where a nonequilibrium spin population has been achieved by using circularly polarized radiation. The characteristics of our observations indicated that the circular photo galvanic effect could be responsible for the generation of the photocurrents. (C) 2009 American Institute of Physics. [doi:10.1063/1.3262496]
\end{abstract}

\section{INTRODUCTION}

Recently, there has been much interest in developing and exploring spin-based devices and phenomena in semiconductors and InSb-based structures, with strong spin-orbit interactions, can play important roles in this direction. Transport measurements have demonstrated mesoscopic spindependent ballistic transport in InSb-based quantum wells (QW). ${ }^{1-3}$ Several signatures of zero field spin splitting in this material system via optical measurements ${ }^{4}$ and, recently, in the beating patterns of the magnetoresistance were observed. ${ }^{5}$ One of the key challenges in developing spinbased devices is to generate, control, and measure spin currents directly. Generation of spin photocurrent was suggested by Averkiev and D'yakonov ${ }^{6}$ and D'yakonov and Perel', and was later observed in bulk n-type $\mathrm{Al}_{0.27} \mathrm{Ga}_{0.73} \mathrm{As}$ at $77 \mathrm{~K}$. The focus of this study was to generate spin-polarized currents in n-type InSb-based films and QWs via the circular photogalvanic effect (CPGE). ${ }^{8-12}$

A simplified picture of CPGE to generate spin-polarized current is presented in Fig. 1. The combination of spin splitting and the optical selection rules can result in an unbalanced occupation of the $k_{+}$and $k_{-}$states and therefore generate a spin-polarized current. It is important to note that the CPGE current decays with the momentum relaxation time and the direction of the current depends on the polarity of the circularly polarized light. The splitting in $k$ space can originate from the bulk inversion asymmetry (BIA), known as the Dresselhaus splitting, ${ }^{13}$ as well as the structural inversion asymmetry (SIA), known as the Rashba term. ${ }^{14}$ The Dresselhaus term is reflected in a $k^{3}$ term and SIA as a $k$ term in the Hamiltonian in the presence of spin-orbit coupling. Averaging the cubic term in two-dimensional (2D) systems along the quantization axis, when only the lowest subband is occupied, ${ }^{11}$ results in a linear term in $k$. In addition, in a 2D system, both BIA and SIA can contribute to the photocurrent with predicted nonequal contributions. ${ }^{15}$ Spin-polarized current can be generated via the spin galvanic effect (SGE), where nonequilibrium distribution of electrons in second

\footnotetext{
${ }^{a)}$ Author to whom correspondence should be addressed. Electronic mail: khoda@vt.edu.
}

conduction band $(\mathrm{CB})$ can relax quickly due to phonon emissions and a current can be caused by asymmetric spin-flip scattering in the first $\mathrm{CB} .{ }^{8}$ The observation of SGE in our structures requires excitation pulses above $20 \mu \mathrm{m}$ that can be only achieved using free electron lasers.

In order to estimate the magnitude of the spin-polarized current originating from CPGE it is easier to consider the scenario where the splitting originates from a $k$ linear term. In this case, the net electron velocity in the $\mathrm{CB}$ is given by ${ }^{8}$

$$
v_{e}=\frac{2\left(\beta m_{h h}-\alpha m_{e}\right)}{\left[\hbar\left(m_{h h}+m_{e}\right)\right]}
$$

where $\beta$ and $\alpha$ are the parameters representing the splitting of the valence and CBs in $k$-space, respectively, and $m_{h h}$ and $m_{e}$ are the heavy hole and electron effective masses. Using suggested values of these parameters, ${ }^{16}$ one can estimate the ratio of the photocurrent due to CPGE in InSb $2 \mathrm{D}$ systems to be larger by more than a factor of 10 compared to GaAs and

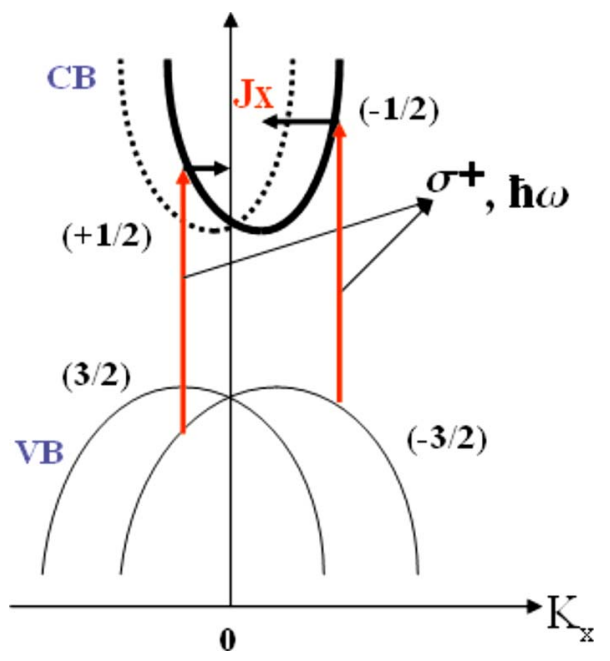

FIG. 1. (Color online) Microscopic picture of CPGE for interband absorption of circularly polarized light with photon energy $\hbar \omega$. When circularly polarized light for a given photon energy induces optical transitions between the VB and the CB, the combination of spin splitting and the optical selection rules can result in an unbalanced occupation of the $k_{+}$and $k_{-}$states generating a spin-polarized current. The current direction depends on the polarization of the incident radiation (Ref. 8). 
TABLE I. The electron density, mobility, and Fermi energy $\left(\mathrm{E}_{\mathrm{F}}\right)$ of the samples at different temperatures.

\begin{tabular}{lcccc}
\hline \hline & $\begin{array}{c}\text { Sample's temperature } \\
(\mathrm{K})\end{array}$ & $\begin{array}{c}\text { Density } \\
\left(\mathrm{cm}^{-3}\right)\end{array}$ & $\begin{array}{c}\text { Mobility } \\
\left(\mathrm{cm}^{2} / \mathrm{V} \mathrm{s}\right)\end{array}$ & $\begin{array}{c}\mathrm{E}_{\mathrm{F}} \\
\mathrm{meV}\end{array}$ \\
\hline InSb/GaAs Film & 300 & $2.6 \times 10^{16}$ & 45500 & 22 \\
& 77 & $6 \times 10^{15}$ & 57000 & 8 \\
InSb/InP Film & 300 & $3.5 \times 10^{16}$ & 2100 & 27.8 \\
& 77 & $1 \times 10^{15}$ & 100 & 2.6 \\
& & & & \\
& Sample's temperature & $\begin{array}{c}\text { Density } \\
\left(\mathrm{cm}^{-2}\right)\end{array}$ & $\begin{array}{c}\text { Mobility } \\
\left(\mathrm{cm}^{2} / \mathrm{V} \mathrm{s}\right)\end{array}$ & $\begin{array}{c}\mathrm{E}_{\mathrm{F}} \\
\mathrm{meV}\end{array}$ \\
& $(\mathrm{K})$ & $6.4 \times 10^{11}$ & 20000 & 109 \\
InSb QW (S360) & 300 & $3.4 \times 10^{11}$ & 61000 & 58 \\
& 77 & $4.9 \times 10^{11}$ & 18000 & 75 \\
InSb QW (S710) & 300 & $8.0 \times 10^{10}$ & 38000 & 12 \\
& 77 & & & \\
\hline \hline
\end{tabular}

InAs systems. This fact is due to larger spin-orbit coupling in InSb, which reflects specifically as a large $\beta$ representing the splitting in the valence band (VB) ${ }^{17}$ In addition, the denominator in Eq. (1) has the smallest value for InSb compared to other III-V semiconductors such as InAs and GaAs. Our measurements demonstrate larger contribution of CPGE to spin-polarized current in InSb-based structures compared to heterostructures such as p-type GaAs/AlGaAs and n-type InAs/AlGaSb with maximum reported currents of 6 and $20 \mathrm{nA} / \mathrm{W}$, respectively. ${ }^{8}$

\section{STRUCTURES AND SETUP}

In this work, we studied two InSb films and two 30-nmwide InSb QWs. The samples were $\sim 5 \times 5 \mathrm{~mm}^{2}$ in size and Table I summarizes their characteristics at different temperatures. The first film, InSb/GaAs, consists of a $0.2 \mu \mathrm{m}$ undoped InSb buffer layer grown on GaAs substrate (100), followed by a $1.3 \mu \mathrm{m}, 2.6 \times 10^{16} \mathrm{~cm}^{-3}$ Te-doped InSb active layer, capped by a $0.05 \mu \mathrm{m}, 1.5 \times 10^{17} \mathrm{~cm}^{-3} \mathrm{Te}$-doped InSb contacting layer. ${ }^{18}$ The second $\mathrm{InSb}$ film, InSb/InP was grown by molecular beam epitaxy with a similar layer structure as the first film. The sample consists of $0.18 \mu \mathrm{m} \mathrm{InSb}$ buffer layer grown on InP (100) substrate following by a $1.2 \mu \mathrm{m}$ Si-doped $\mathrm{InSb}$ active layer capped by a $40 \mathrm{~nm}$ layer of Si-doped InSb. The lattice constant of $\operatorname{InP}(5.868 \AA)$ is closer to that of $\operatorname{InSb}(6.47 \AA)$ than the lattice constant of GaAs (5.654 ̊).

The InSb QWs studied were grown on (100) GaAs and were 30-nm-wide. $\mathrm{S} 360$ has $\sim 35 \mathrm{~nm}$ of $\mathrm{Al}_{x} \mathrm{In}_{1-x} \mathrm{Sb}$ and a single $\mathrm{Si}-\delta$-doped layer above the InSb layer; whereas, S710 has 210 -nm-thick $\mathrm{Al}_{x} \mathrm{In}_{1-x} \mathrm{Sb}$ and three $\delta$-doped layers above the well. The detailed growth conditions of the InSb QW samples were described previously. ${ }^{4,19}$ In a 30 -nm-wide asymmetric $\mathrm{InSb} / \mathrm{Al}_{0.09} \mathrm{In}_{0.91} \mathrm{Sb} \mathrm{QW}$ such as $\mathrm{S} 360$, a doping density of $\sim 2.0 \times 10^{11} \mathrm{~cm}^{-2}$ can result in a built electric field of $3.3 \times 10^{6} \mathrm{~V} / \mathrm{m}$ corresponding to an effective magnetic field of $\sim 0.1 \mathrm{mT} .^{20}$

In general, bulk semiconductors with zinc blende lattice structures are nongyrotropic and CPGE is considered to be forbidden; however, the structures can become gyrotropic in the presence of uniaxial strain or quantum confinement. ${ }^{8,12,17}$

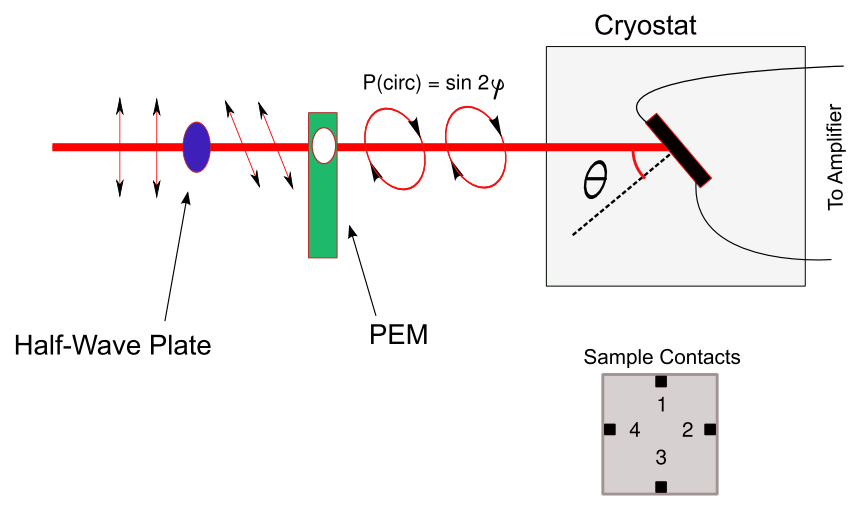

FIG. 2. (Color online) A diagram of the experimental setup. The polarization of the incident radiation, before entering the PEM, rotates by $45^{\circ}$ using a half-wave plate. The PEM modulates the polarization of the radiation and the helicity of the output varies as $\sin 2 \varphi$, where $\varphi$ is the phase angle between the $\mathrm{x}$ and $\mathrm{y}$ components of the radiation's electric field. The beam is then focused on the sample at an angle $\theta$. The lower sketch shows the contacts' configuration.

Therefore, CPGE can contribute to the generation of spinpolarized currents in QWs. To probe a possible contribution of strain to CPGE in the InSb films, we monitored $x$-ray reflections from (004) and (335) planes of the InSb/GaAs film. From these measurements, we could deduce the out-ofplane lattice constant $(c=6.4832 \pm 0.0021 \AA)$ and the average in-plane lattice constant $\left(a_{L}=6.4756 \pm 0.0023 \AA\right)$. If the InSb layer was fully relaxed, $a_{L}$ and $\mathrm{c}$ would both be $6.4794 \AA$. If the InSb layer was fully strained on the GaAs substrate, $a_{L}$ and $c$ would be 5.6533 and $7.3041 \AA$, respectively. Since $c$ is only slightly larger than $a_{L}$ in this sample, the layer is only slightly strained. From the usual definition of percentage relaxation, $R=(6.4756-5.6533) /(6.4797$ $-5.6533)$, the film is $99.54 \%$ relaxed. A similar result is expected for the InSb/InP film. The lack of strain and the observation of CPGE in these films are suggesting other mechanisms responsible for the generation of the spinpolarized currents in nongyrotropic structures.

We used laser pulses from a Ti:sapphire laser (100 fs pulse width and $80 \mathrm{MHz}$ repetition rate) with tunable wavelengths from $750-800 \mathrm{~nm}$ with an average power of $\sim 140 \mathrm{~mW}$ corresponding to energy per pulse of $\sim 2 \mathrm{~nJ}$. Our measurements were mainly performed at $800 \mathrm{~nm}$, where the photon energy is far above the bandgap of InSb. Although this configuration might not be the most efficient pumping scheme, it allowed us to use a photoelastic modulator (PEM) with an $80 \mathrm{MHz}$ system. A spot size of $\sim 1 \mathrm{~mm}$ corresponding to a power density of $\sim 18 \mathrm{~W} / \mathrm{cm}^{2}$ transforming to a photoinduced density of $\sim 6 \times 10^{15} \mathrm{~cm}^{-3}$. As shown in Fig. 2, the sample's angle, $\theta$ with respect to the direction of the incident beam, was adjusted to optimize the amount of the spin-polarized current. The laser beam was passed through a half-wave plate to rotate the plane of polarization and then passed through a PEM. The modulation frequency of our PEM was set at $50 \mathrm{kHz}$, allowing detection of the voltage across the contacts using a lock-in amplifier. The measured voltages were converted to the currents using the resistance of the samples from a four-contact measurement. 


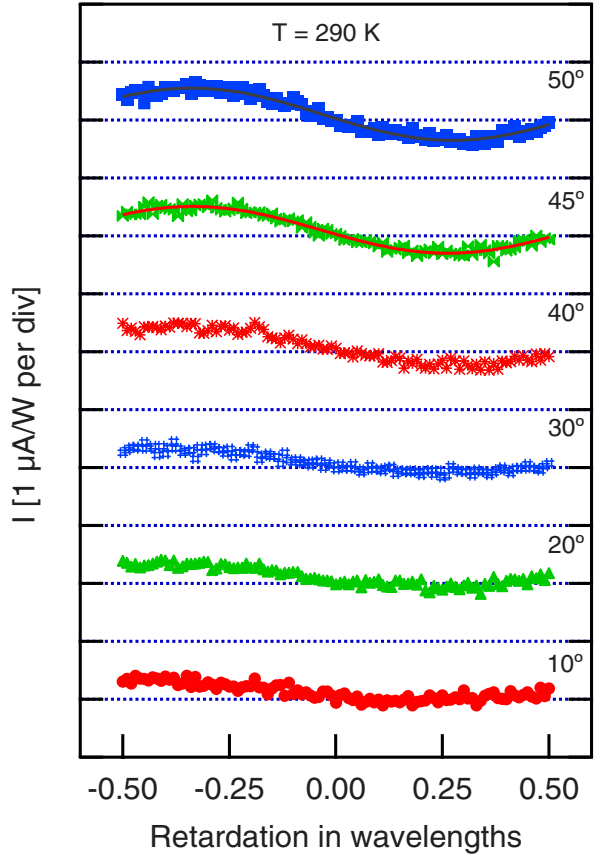

FIG. 3. (Color online) Traces of the photoinduced current at $290 \mathrm{~K}$ in $\mathrm{InSb} / \mathrm{GaAs}$ film for several $\theta$ as a function of retardation with step size of $0.01 \lambda$. The voltages from $[2 \rightarrow 4]$ were measured and converted into the current. The magnitude of the current $\sim 1 \mu \mathrm{A} / \mathrm{W}$ at $\theta=50^{\circ}$, tailing off to a very small signal at $\theta=10^{\circ}$.

\section{OBSERVATIONS AND DISCUSSIONS}

Examples of our measurements on InSb films and QWs are presented here. Figure 3 shows the currents at $290 \mathrm{~K}$ in $\mathrm{InSb} / \mathrm{GaAs}$ film, for several $\theta$ as a function of retardation. As shown in Fig. 1, the electronic transition selection rules demonstrate the sensitivity of CPGE to the helicity of the incident radiation. The helicity can be expressed as

$$
P_{\text {circ }}=\frac{I_{\sigma_{+}}-I_{\sigma_{-}}}{I_{\sigma_{+}}+I_{\sigma_{-}}}=\sin 2 \varphi .
$$

$P_{\text {circ }}$ can vary from -1 to +1 for $\sigma_{-}$and $\sigma_{+}$polarization, respectively, and $\varphi$ is the phase angle between the $\mathrm{x}$ and $\mathrm{y}$ components of the light electric field. Therefore, as shown in Fig. 2, the variation in the wavelength retardation can result in a period of $\pi$ in the current. The current direction changes sign depends on the polarization of the incident radiation.

As shown in Fig. 3, the magnitude of the current is maximum at $\theta=50^{\circ}$, tailing off from $\sim 1 \mu \mathrm{A} / \mathrm{W}$ to zero below $\theta=10^{\circ}$. An asymmetry pattern is evident in all traces, which seems to be consistent throughout the measurements in both films and the InSb QW. This fact can be attributed to the quality of the contacts. Unlike four-contact measurements, in a two-contact measurement poor contacts can act as a Schottky barrier, resulting in rectification of the induced current. Two rectifying contacts would result in a very small photocurrent, while one Ohmic and one rectifying contact would appear as a significant difference in the induced current between two contacts.

This fact was tested and demonstrated in Fig. 4(a), by reversing the connections to a specific pair, the polarity of the voltage changes but clearly the size of the voltage is
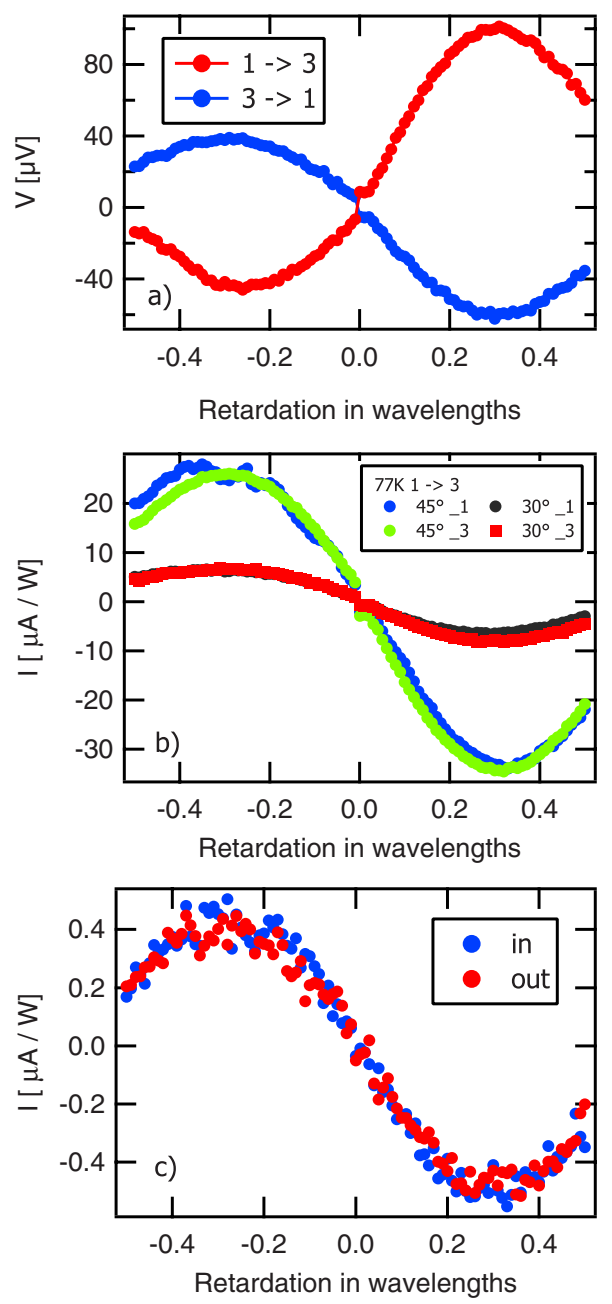

FIG. 4. (Color online) (a) Probe of the asymmetry in the traces and the contacts' quality. Measurements were performed on the InSb/GaAs film at $\mathrm{T}=290 \mathrm{~K}$ and $\theta=45^{\circ}$. Dependence of the asymmetry on the direction (rectification). The traces presented here are indicating that the asymmetric pattern is independent of direction; rather, positive retardation $(\varphi>0)$ generates a larger signal than negative retardation $(\varphi<0)$. (b) Repeatability of the measurements with changing the contacts' configurations: a series of measurements were performed by interchanging horizontal and vertical contacts resulting in reproducible measurements. (c) To probe possible birefringence effects from the cryostat's window: traces with and without the window were taken resulting in identical traces.

independent of direction for $(\varphi<0)$; instead, the observed asymmetry for positive retardation $(\varphi>0)$ is much larger than for negative $(\varphi<0)$. Several trials with newly soldered and annealed contacts mitigated some of the differences; however, the observed asymmetry could not be eliminated entirely by improvements to the contacts' quality. In a study by Yang et al., ${ }^{10}$ an asymmetry in the measured spinpolarized photocurrent was also evident. The magnitude of the current in one of their samples (sample E) for the negative retardation was smaller than for the positive retardation.

Figure 4(b) demonstrates the reproducibility of the measurements for $\theta=45^{\circ}$ and $\theta=30^{\circ}$ at $77 \mathrm{~K}$. The contacts' configuration were switched back and forth across the sample from $[1 \rightarrow 3]$ to $[2 \rightarrow 4]$, and identical traces were measured in each case. In addition, we examined any possible contribution from the cryostat's window (fused silica) to the signals. As shown in Fig. 4(c) at $\mathrm{T}=290 \mathrm{~K}$ and $\theta=45^{\circ}$, two 

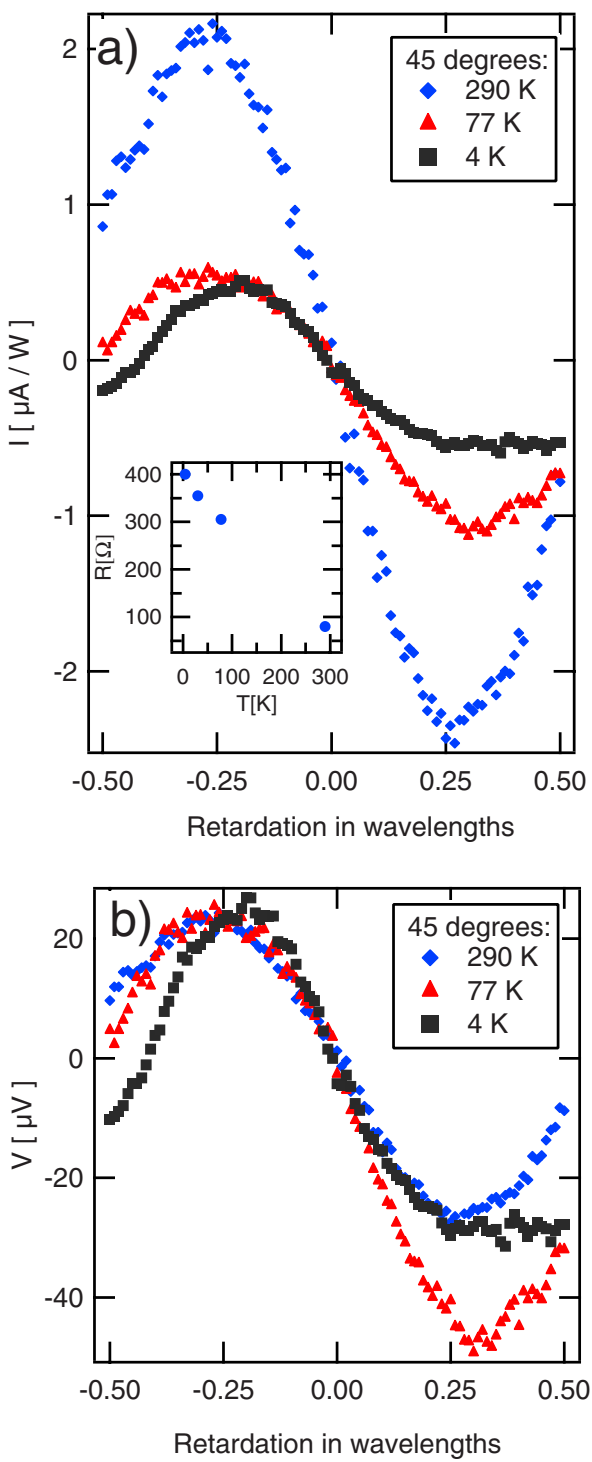

FIG. 5. (Color online) (a) The temperature dependence of photoinduced currents. The inset shows the sample resistance obtained from a four-contact measurement. (b) Photoinduced voltages were used to calculate the currents presented in Fig. 5(a). Our measurements demonstrate photoinduced currents with a maximum of about $2 \mu \mathrm{A} / \mathrm{W}$ at $290 \mathrm{~K}$ for $[2 \rightarrow 4]$ pins configuration.

measurements were taken with and without the window for $[1 \rightarrow 3]$ pins configuration. The traces appear nearly identical, indicating no birefringence effect induced by the window.

Examples of the temperature dependence currents in $\mathrm{InSb} / \mathrm{GaAs}$ film for $\theta=45^{\circ}$ are plotted in Fig. 5(a). As shown in Fig. 5(b), in order to calculate the currents, the measured voltages across the samples and the sample's resistance (obtained from a four-contact measurement) were used. The asymmetry in the traces is more significant at $4 \mathrm{~K}$; however, the maximum voltage at $-\lambda / 4$ for all the traces is almost identical. In addition, for a given laser fluence, we observe strong dependence of the photocurrent to the orientation of the contacts. This fact can be realized by comparing the magnitude of the current at $77 \mathrm{~K}$ in Fig. 4(b) for $[1 \rightarrow 3]$ configuration and the measurements presented in Fig. 5(a) for [2 $\rightarrow 4]$.
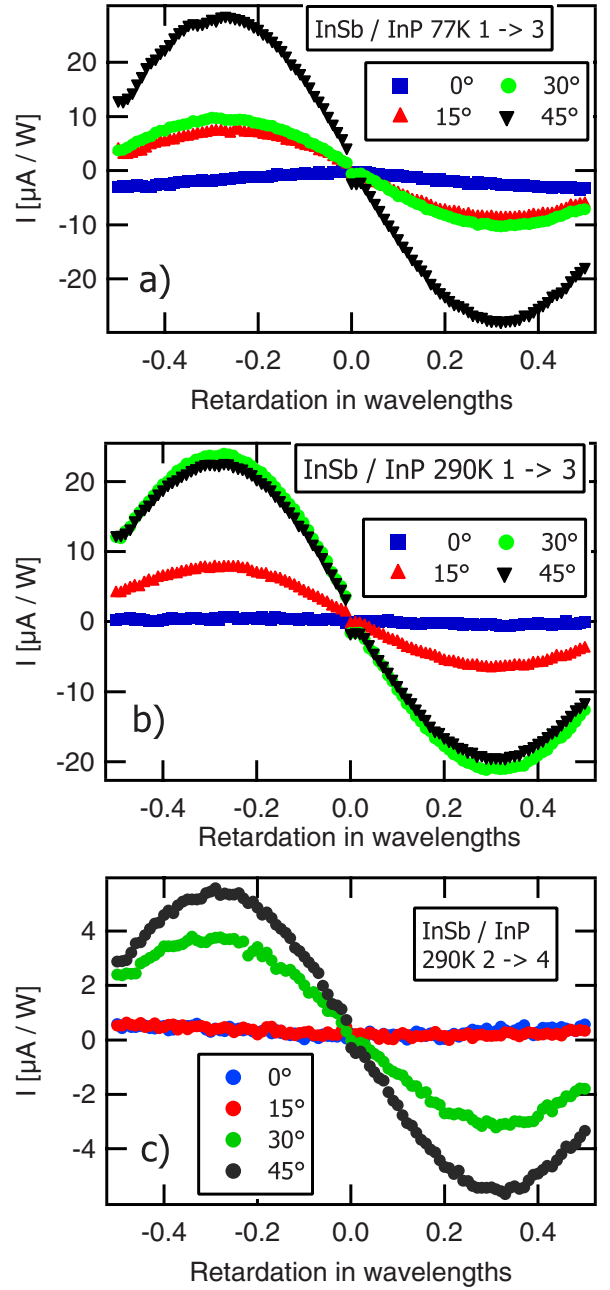

FIG. 6. (Color online) Photoinduced currents in InSb grown on InP substrate at (a) 77 and (b) $290 \mathrm{~K}$. The maximum signal occurs at a $\theta=45^{\circ}$ tailing to zero at smaller incident angles. (c) Using contacts perpendicular to those in (a) and (b), the observed currents are weaker, consistent with the observation in $\mathrm{InSb} / \mathrm{GaAs}$ film.

The observation in the InSb/GaAs film, motivated us to grow the second InSb film on InP substrate. A series of measurements on an InSb/InP film at 77 and $290 \mathrm{~K}$ resulted in similar observations as the first film. Figure 6 demonstrates examples of these measurements for two different contact configurations as a function of retardation. The maximum current (converted from the voltage measurements) at 290 and $77 \mathrm{~K}$ at $\theta=45^{\circ}$ for $[1 \rightarrow 3]$ configuration is $\sim 20 \mu \mathrm{A} / \mathrm{W}$, comparable to the measurements on the $\mathrm{InSb} / \mathrm{GaAs}$ film at 77 K. As shown in Figs. 6(a) and 6(b), the currents for the $[1 \rightarrow 3]$ configuration are larger compared to the measurements for $[2 \rightarrow 4]$, presented in Fig. 6(c). Not shown here, the magnitude of the current for $[2 \rightarrow 4]$ in the InSb/InP film at $77 \mathrm{~K}$ increased by about a factor of 2 compared to the measurements at $290 \mathrm{~K}$. The asymmetry in the traces and the large differences in the signal with respect to contacts' directions are similar to those seen in the InSb/GaAs film.

The natural extension of our studies was to probe $\mathrm{InSb}$ QWs. Figure 7 demonstrates the generation of spin-polarized currents in an asymmetric InSb QW (S360) at $77 \mathrm{~K}$ for $[1$ $\rightarrow 3$ ] configuration. The signal falls to zero at $\theta=30^{\circ}$ from a maximum at $\theta=40^{\circ}$. Symmetry analysis shows that the 


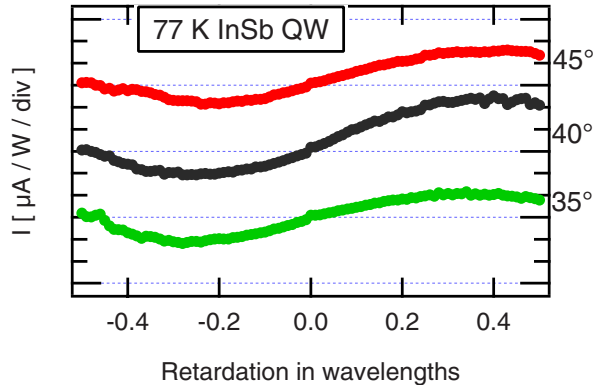

FIG. 7. (Color online) CPGE effect in an InSb QW at $77 \mathrm{~K}$. The maximum signal was measured at $\theta=40^{\circ}$, approaching zero at $\theta=30^{\circ}$. The currents were observed only for $[1 \rightarrow 3]$ configuration. From the helicity-dependent photocurrent in (001) QW structures one expects to observe CPGE only at oblique incident radiation and in the zinc-blende-type materials, only in the plane of QWs.

helicity-dependent photocurrent in (001) QW structures can be induced only at oblique incident radiation and in the zincblende-type structures resulting in an electric current in the plane of QWs. ${ }^{8,21,22}$ This fact could explain the absence of current for the $[2 \rightarrow 4]$ pin configuration in this QW. Unlike the InSb-based films and several other reported measurements, ${ }^{8}$ no photocurrent was observed at room temperature in S360. Under the same experimental conditions for $\mathrm{S} 360$, a series of measurements on two different pieces of the symmetric sample (S710) at different temperatures and angles of laser incidence $\theta$, suggested the absence of any spin-polarized current. This fact could be due to the lack of asymmetry in the confinement potential of S710 or the thicker layer above the well in S710 compared to S360. The absorption length in these heterostructures (in the wavelength region used in this study) is about $0.5 \mu \mathrm{m}$; therefore, the excitation wavelength can create carriers in the $\mathrm{Al}_{x} \mathrm{In}_{1-x} \mathrm{Sb}$ barrier layer as well as in the QWs. An earlier measurement on an undoped InSb MQW demonstrated the generation of high density photoexcited carriers in the wells using $800 \mathrm{~nm}$ pulses. Selective probe of the CB demonstrated carrier relaxation time of $\sim 200 \mathrm{ps}^{23}$

In summary, we demonstrate the generation of spinpolarized currents in InSb-based structures with the signatures similar to the spin-polarized photocurrents originated from the CPGE. In the absence of a significant strain, our observations in the InSb films cannot be explained by the existing theoretical models. ${ }^{8,17}$ The lack of spin-polarized photocurrent in the symmetric InSb QWs will be probed further, when the samples with similar layer structures as the asymmetric ones, become available.

\section{ACKNOWLEDGMENTS}

Supported by NSF Grant Nos. NSF-DMR-0507866, NSF-DMR-0603752, NSF-DMR-0520550, and NSF-DMR0618235, and AFOSR Young Investigator Program Grant No. 06NE231.

${ }^{1}$ N. Goel, J. Graham, J. C. Keay, K. Suzuki, S. Miyashita, M. B. Santos, and Y. Hirayama, Physica E (Amsterdam) 26, 455 (2005).

${ }^{2}$ H. Chen, J. J. Heremans, J. A. Peters, N. Goel, S. J. Chung, and M. B. Santos, Appl. Phys. Lett. 86, 032113 (2005).

${ }^{3}$ H. Chen, J. J. Heremans, J. A. Peters, N. Goel, S. J. Chung, and M. B. Santos, Appl. Phys. Lett. 84, 5380 (2004).

${ }^{4}$ G. A. Khodaparast, R. E. Doezema, S. J. Chung, K. J. Goldammer, and M. B. Santos, Phys. Rev. B 70, 155322 (2004).

${ }^{5}$ A. M. Gilbertson, W. R. Branford, M. Fearn, L. Buckle, P. D. Buckle, T. Ashley, and L. F. Cohen, Phys. Rev. B 79, 235333 (2009).

${ }^{6}$ N. S. Averkiev and M. I. D’yakonov, Fiz. Tekh. Poluprovodn. 17, 629 (1983) [Sov. Phys. Semicond. 17, 393 (1983)].

${ }^{7}$ M. I. D'yakonov and V. I. Perel', Pis'ma Zh. Eksp. Teor. Fiz. 13, 206 (1971) [Sov. Phys. JETP 13, 144 (1971)].

${ }^{8}$ S. D. Ganichev, E. L. Ivchenko, S. N. Danilov, J. Eroms, W. Wegscheider, D. Weiss, and W. Prettl, Phys. Rev. Lett. 86, 4358 (2001); S. D. Ganichev and W. Prettl, J. Phys.: Condens. Matter 15, R935 (2003).

${ }^{9}$ V. V. Bel'kov, S. D. Ganichev, P. Schneider, C. Back, M. Oestreich, J. Rudolph, D. Hägele, L. E. Golub, W. Wegscheider, and W. Prettl, Solid State Commun. 128, 283 (2003).

${ }^{10}$ C. L. Yang, H. T. He, L. Ding, L. J. Cui, Y. P. Zeng, J. N. Wang, and W. K. Ge, Phys. Rev. Lett. 96, 186605 (2006).

${ }^{11}$ J. Zhou, J. L. Cheng, and M. W. Wu, Phys. Rev. B 75, 045305 (2007).

${ }^{12}$ Z. Zhang, R. Zhang, Z. L. Xie, B. Liu, M. Li, D. Y. Fu, H. N. Fang, X. Q. Xiu, H. Lu, Y. D. Zhenga, Y. H. Chen, C. G. Tang, and Z. G. Wang, Solid State Commun. 149, 1004 (2009).

${ }^{13}$ G. F. Dresselhaus, Phys. Rev. 100, 580 (1955).

${ }^{14}$ Y. A. Bychkov and E. I. Rashba, J. Phys. C 17, 6039 (1984); Y. A. Bychkov and E. E. Rashba, Pis'ma Zh. Eksp. Teor. Fiz. 39, 66 (1984) [JETP Lett. 39, 78 (1984)].

${ }^{15}$ L. E. Golub, Phys. Rev. B 67, 235320 (2003).

${ }^{16} \mathrm{R}$. Winkler, Spin-Orbit Coupling Effects in Two-Dimensional Electron and Hole Systems, Springer Tracts in Modern Physics (Springer, Berlin, 2003), Vol. 191, p. 216.

${ }^{17} \mathrm{R}$. Winkler, in Handbook of Magnetism and Advanced Magnetic Materials, edited by H. Kronmuller and S. Parkin (Wiley, Chichester, 2007), Vol. 5, p. 2830.

${ }^{18}$ M. W. Pelczynski, J. J. Heremans, and S. Schwed, Mater. Res. Soc. Symp. Proc. 607, 65 (2000).

${ }^{19}$ K. J. Goldammer, W. K. Liu, G. A. Khodaparast, S. C. Lindstrom, M. B. Johnson, R. E. Doezema, and M. B. Santos, J. Vac. Sci. Technol. B 16, 1367 (1998).

${ }^{20}$ P. Pfeffer and W. Zawadzki, Phys. Rev. B 68, 035315 (2003).

${ }^{21}$ S. A. Tarasenko, JETP Lett. 85, $182(2007)$.

${ }^{22}$ S. Giglberger, L. E. Golub, V. V. Belkov, S. N. Danilov, D. Schuh, C. Gerl, F. Rohlfing, J. Stahl, W. Wegscheider, D. Weiss, W. Prettl, and S. D. Ganichev, Phys. Rev. B 75, 035327 (2007).

${ }^{23}$ G. A. Khodaparast, D. C. Larrabee, J. Kono, D. S. King, S. J. Chung, and M. B. Santos, Phys. Rev. B 67, 035307 (2003). 